PROCEEDINGS OF THE

AMERICAN MATHEMATICAL SOCIETY

Volume 129, Number 6, Pages 1763-1767

S 0002-9939(00)05685-9

Article electronically published on December 13, 2000

\title{
EXEMPLES SÉPARANT CERTAINES CLASSES D'ALGÈBRES TOPOLOGIQUES
}

\author{
Z. ABDELALI AND M. CHIDAMI
}

(Communicated by Dale Alspach)

\begin{abstract}
Esterle (1979) and Żelazko (1996 and 1990) gave an example of an algebra which cannot be topologized as a topological algebra (with jointly continuous multiplication), and Müller (in 1991) showed that there exists a topological algebra, which is indeed a locally bounded algebra, which cannot be topologized as a locally convex algebra.

To complete this study on the separation between the classes of algebras, we construct for every $p \in] 0,1]$ a locally bounded algebra which is $q$-normed for every $q \in] 0, \frac{p}{p+2}$ [ which cannot be topologized as a locally $p$-convex algebra, and we deduce an example of a locally pseudoconvex algebra which cannot be topologized as a locally $p$-convex algebra for every $p \in] 0,1]$. We also show the existence of a topological algebra which cannot be topologized as a locally pseudoconvex algebra.
\end{abstract}

Tous les espaces vectoriels et les algèbres considérés ici sont réels ou complexes. Une algèbre topologique $A$ est un espace vectoriel topologique séparé muni d'une multiplication associative simultanément continue. On dira que l'algèbre $A$ est localement bornée, localement pseudoconvexe, ... si l'espace vectoriel topologique sous-jacent possède la même propriété. Un espace vectoriel topologique séparé $X$ est dit localement borné ou quasi-normé si la topologie de $X$ est définie par une quasi-norme $f$, c'est à dire que $f$ est une application sur $X$ non négative qui ne s'annule qu'en zéro, telle que pour un certain $p \in] 0,1]$, pour tout scalaire $\lambda$ et tout $(x, y) \in X^{2}, f(x+y) \leq 2^{\frac{1}{p}}(f(x)+f(y))$ et $f(\lambda x)=|\lambda| f(x)$, dans ce cas le sous-ensemble $V=\{x \in X / f(x) \leq 1\}$, qui est un voisinage borné de zéro, vérifie $V+V \leq 2^{\frac{1}{p}+1} V$, ainsi la topologie de $X$ peut être définie par une $q$-norme pour tout $q \in] 0, \frac{p}{p+1}[([\underline{3}$, Theo.1, $\S 2$, Chap.III] $)$, rappelons qu'une $q$-semi-norme \|\| sur l'espace vectoriel $X$ est une application non négative, sous additive et vérifiant pour tout $x \in X$ et tout scalaire $\lambda,\|\lambda x\|=|\lambda|^{q}\|x\|$, si on a en plus $x=0$ si $\|x\|=0$ on dira que \|\| est une $q$-norme. De plus si $(A, f)$ est une algèbre topologique quasi-normée alors pour tout $q \in] 0, \frac{p}{p+1}$ [ la topologie de $A$ peut être définie par une $q$-norme sous-multiplicative.

L'espace $X$ est dit localement $p$-convexe, $0<p \leq 1$, (resp. localement pseudoconvexe) si la topologie de $X$ est définie par une famille de $p$-semi-normes (resp. $p_{i}$-semi-normes $\left.\left.p_{i} \in\right] 0,1\right], i \in I$ ). Remarquons que pour toute $p$-semi-norme \|\| , l'ensemble absorbant $V=\{x \in X /\|x\| \leq 1\}$ est un $p$-disque, c'est à dire, pour tout

Received by the editors December 11, 1998 and, in revised form, May 24, 1999 and October 1, 1999.

1991 Mathematics Subject Classification. Primary 46J05; Secondary 46J40. 
couple de scalaires $(\lambda, \beta),|\lambda|^{p}+|\beta|^{p} \leq 1, \lambda V+\beta V \subseteq V$ (il est clair que pour tout $q \leq p$, un $p$-disque est un $q$-disque). Ainsi, pour qu'une algèbre $A$ soit topologisable comme une algèbre localement $p$-convexe (resp. localement pseudoconvexe) il faut et il suffit qu'il existe sur $A$ une famille de $p$-disques (resp. $p_{i}$-disques, $\left.\left.p_{i} \in\right] 0,1\right]$ ) absorbants $\left(V_{i}\right)_{i \in I}$ vérifiant $\bigcap_{i \in I} V_{i}=\{0\}$ et pour tout $i \in I$, il existe $j_{i} \in I$ tel que $V_{j_{i}}+V_{j_{i}} \subseteq V_{i}$ et $V_{j_{i}} V_{j_{i}} \subseteq V_{i}$. Rappelons qu'un espace vectoriel topologique séparé $X$ est métrisable si est seulement si $X$ est F-normé (une F-norme \| \| est une application non négative, sous additive et vérifiant pour tous $x$ et $x_{n} \rightarrow 0$ dans $X$ et tous scalaires $\lambda$ et $\lambda_{n} \rightarrow 0,\left\|\lambda x_{n}\right\| \rightarrow 0$ et $\left\|\lambda_{n} x\right\| \rightarrow 0$ et si $\left.|\lambda| \leq 1,\|\lambda x\| \leq\|x\|\right)$.

Soit $A$ un espace vectoriel ou une algèbre, pour tout $(p, q), 0<q<p \leq 1$, si A est $p$-normé (resp. localement $p$-convexe), alors $A$ est $q$-normé (resp. localement $q$ convexe), et dans tous ces cas $A$ est localement pseudoconvexe. Pour les définitions et les propriétés de ces classes d'espaces et d'algèbres topologiques voir par exemple S. Rolewicz [3], S. Simmons [4], ou le travail de P. Turpin [5] qui contient plus de détail ainsi que des exemples montrant que les inclusions entre ces classes sont strictes.

Le problème de la comparaison entre les classes d'algèbres sous-jacentes à ces classes d'algèbres topologiques (autrement dit, étant donné deux classes d'algèbres topologiques, existe-t-il une algèbre de la classe la plus large qui est non topologisable comme une algèbre de l'autre classe), donne un cadre naturel aux trvaux, de Żelazko [6] et [7], et Müller [2], dont on trouve, d'une part, des exemples d'algèbres qui ne peuvent pas être des algèbres topologiques, d'autre part on trouve, dans [2], un exemple d'algèbre topologique, qui est en fait une algèbre localement bornée, qui ne peut être munie d'aucune topologie d'algèbre localement-convexe.

Ici, en donnant un lemme modifiant, pour le nécessaire, des techniques du théorème 2 [2], nous complétons ces travaux en montrant (corollaire 1) que pour tout $p \in] 0,1]$, il existe une algèbre localement bornée ( $q$-normée pour tout $q \in] 0, \frac{p}{p+2}[$ ) complète ne pouvant être munie d'aucune topologie d'algèbre localement $p$-convexe.

Ainsi, nous déduisons dans le corollaire 2 , un exemple d'algèbre localement pseudoconvexe métrisable complète qui ne peut être munie d'aucune topologie d'algèbre localement $p$-convexe pour tout $p \in] 0,1]$.

Finalement, nous donnons un théorème qui montre l'existence d'une algèbre topologique métrisable complète ne pouvant être munie d'aucune topologie d'algèbre localement pseudoconvexe.

Lemme. Pour tout $p \in] 0,1]$, il existe une algèbre commutative, localement bornée (q-normée pour tout $q \in] 0, \frac{p}{p+2}[) A_{p}$ et un élément non nul $c_{p} \in A_{p}$, tels que:

1. $c_{p} \cdot A_{p}=\{0\}$;

2. si $V$ et $W$ sont deux $p$-disques absorbants tels que $V . V \subseteq W, c_{p} \in W$.

Preuve. Sauf quelques modifications nécessaires la preuve est similaire à celle du théorème $2[2]$.

Soient $K$ un ensemble non dénombrable et $D$ l'ensemble $\mathbf{N}^{\mathbf{N} \times K}$ ( $\mathbf{N}$ désigne l'ensemble des nombres entiers positifs non nuls). Pour tout $d \in D$ et tout $(n, k) \in$ $\mathbf{N} \times K, d_{n k}$ désignera l'image de $(n, k)$ par $d$.

Pour tout $d \in D$ et $n \in \mathbf{N}$, il existe un sous-ensemble $K_{d n}$ de $K$ et un élément $d_{n}$ de $\mathbf{N}$, tels que $\operatorname{card}\left(K_{d n}\right)=d_{n}$ et pour tout $k \in K_{d n}, d_{n}=d_{n k}$.

\footnotetext{
${ }^{1}$ L'exemple [7] peut être déduit du corollaire 2.2 [1] d'Esterle.
} 
Soit $A$ l'espace vectoriel engendré par les symboles : $c, x_{n k}((n, k) \in \mathbf{N} \times K)$, $a_{d}(d \in D)$ et $y_{d n k}\left(d \in D, n \in \mathbf{N}, k \in K_{d n} \subseteq K\right)$.

On munit $A$ du produit commutatif défini par:

$$
\begin{gathered}
c A=y_{d n k} A=\{0\}\left(d \in D, n \in \mathbf{N}, k \in K_{d n}\right), \\
a_{d} a_{d^{\prime}}=0\left(\left(d, d^{\prime}\right) \in D\right), \\
x_{n k} \cdot x_{n^{\prime} k^{\prime}}=0\left(\left(n, n^{\prime}\right) \in \mathbf{N}^{2},\left(k, k^{\prime}\right) \in K^{2}\right), \\
x_{n k} \cdot a_{d}=\left\{\begin{array}{lll}
\left(d_{n}\right)^{\frac{1}{p}} y_{d n k} & \text { si } & d \in D, n \in \mathbf{N} \text { et } k \in K_{d n}, \\
0 & \text { si } & k \notin K_{d n} .
\end{array}\right.
\end{gathered}
$$

Le produit de trois éléments quelconques est nul, ainsi $A$ est une algèbre.

Soit $L$ l'ensemble des fonctions scalaires $\lambda: k \rightarrow \lambda_{k}$ définies sur $K$ à support fini. D'après le lemme 3 [2], il existe sur $L$ une suite de fonctions réels non négatives $\left(m_{n}\right)_{n+1 \in \mathbf{N}}$ vérifiant $m_{i+j}(\lambda+\beta) \leq m_{i}(\lambda)+m_{j}(\beta), m_{i}(\lambda) \leq m_{j}(\lambda)$ pour $j \leq i$ et $m_{n_{\lambda}}(\lambda)=0$ où $n_{\lambda}=\operatorname{card}\left(\left\{k \in K / \lambda_{k} \neq 0\right\}\right.$ ) (on aura besoin de la définition et les propriétés de $\left(m_{n}\right)_{n+1 \in \mathbf{N}}$ données dans le lemme 3 [2]).

Soit $h$ l'application définie sur $L$ par:

$$
h(\lambda)=\sum_{i=0}^{\infty} u_{i} m_{i}(\lambda) \text { où } u_{i}=(i+1)^{\frac{2}{p}}-(i)^{\frac{2}{p}} \text { on a } \frac{u_{2 i+1}+u_{2 i}}{u_{i}}=2^{\frac{2}{p}} .
$$

L'application $h$ vérifie $h(\lambda+\beta) \leq 2^{\frac{2}{p}}(h(\lambda)+h(\beta))$. En effet:

$$
\begin{aligned}
& h(\lambda+\beta)=\sum_{i=0}^{\infty} u_{2 i} m_{i+i}(\lambda+\beta)+\sum_{i=0}^{\infty} u_{2 i+1} m_{i+(i+1)}(\lambda+\beta) \\
& \leq \sum_{i=0}^{\infty} u_{2 i}\left(m_{i}(\lambda)+m_{i}(\beta)\right)+\sum_{i=0}^{\infty} u_{2 i+1}\left(m_{i}(\lambda)+m_{i+1}(\beta)\right) \\
& \leq \sum_{i=0}^{\infty}\left(u_{2 i}+u_{2 i+1}\right)\left(m_{i}(\lambda)+m_{i}(\beta)\right) \leq 2^{\frac{2}{p}}(h(\lambda)+h(\beta)) .
\end{aligned}
$$

Soit $f$ la quasi-norme définie sur $A$ par: pour tout $u \in A$ tel que

$$
\begin{gathered}
u=\alpha c+\sum_{n \in N} \sum_{k \in K} \beta_{n k} x_{n k}+\sum_{d \in D} \gamma_{d} a_{d}+\sum_{d \in D} \sum_{n \in \mathbf{N}} \sum_{k \in K_{d n}} \delta_{d n k} y_{d n k}, \\
f(u)=|\alpha|+\sum_{n \in N} h\left(\left\{\beta_{n k}\right\}_{k \in K}\right)+\sum_{d \in D}\left|\gamma_{d}\right|+\sum_{d \in D} \sum_{n \in \mathbf{N}}\left(\frac{1}{d_{n}}\right)^{\frac{1}{p}} h\left(\left\{\delta_{d n k}\right\}_{k \in K}\right)
\end{gathered}
$$

en posant $\delta_{d n k}=0$, pour $k \in K \backslash K_{d n}$.

D'une manière analogue à [2] on montre que la quasi-norme $f$ vérifie:

- $u=0$ si et seulement si $f(u)=0, f(\lambda u)=|\lambda| f(u)$;

- $f(u+v) \leq 2^{\frac{2}{p}}(f(u)+f(v))$;

- $f(u v) \leq 2^{\frac{2}{p}} f(u) f(v)$. 
Ainsi, l'algèbre localement bornée $(A, f)$ est $q$-normée pour tout $q \in] 0, \frac{p}{p+2}[$.

Soit $M$ le sous-espace vectoriel de $A$ engendré par l'ensemble:

$$
\left\{c-\left(\frac{1}{d_{n}}\right)^{\frac{1}{p}} \sum_{k \in K_{d n}} y_{d n k} /(d, n) \in D \times \mathbf{N}\right\}
$$

l'espace $M$ est un idéal et si $(d, n) \in D \times \mathbf{N}, f\left(\left(\frac{1}{d_{n}}\right)^{\frac{1}{p}} \sum_{k \in K_{d n}} y_{d k n}\right)=1$, ainsi on montre que $M$ est fermé exactement comme dans le lemme 3 [2]. Soit $A_{p}$ l'algèbre topologique quotient $A / M$ et soit $c_{p}$ l'image de $c$ par la surjection canonique, il est clair que $c_{p} \neq 0$.

La technique utiliser dans la preuve du lemme 5 [2], nous permet de déduire la deuxième assertion de notre lemme, la première assertion étant évidente.

L'algèbre complétée de $A_{p}$ nous permet de déduire le résultat suivant:

Corollaire 1. Pour tout $p \in] 0,1]$, il existe une algèbre commutative localement bornée (q-normée pour tout $q \in] 0, \frac{p}{p+2}[$ ) complète ne pouvant être munie d'aucune topologie d'algèbre localement p-convexe.

Corollaire 2. Il existe une algèbre commutative localement pseudoconvexe métrisable complète ne pouvant être munie d'aucune topologie d'algèbre localement pconvexe pour tout $p \in] 0,1]$.

Preuve. Soit $A_{n}(n \in \mathbf{N})$ une algèbre vérifiant les conditions du corollaire 1, pour $p=\frac{1}{n}$, l'algèbre topologique produit $\prod_{n \in \mathbf{N}} A_{n}$ répond à la question.

Théorème. Il existe une algèbre topologique métrisable complète commutative ne peuvant être munie d'aucune topologie d'algèbre localement pseudoconvexe.

Preuve. Soit $\left(p_{n}\right)_{n \geq 0}$ la suite d'éléments de ]0,1] définie par:

$$
p_{0}=1 \text { et } p_{n+1}=\frac{p_{n}}{2\left(p_{n}+2\right)} \text {. }
$$

Pour tout $n \in \mathbf{N}$, soit $A_{n}$ une algèbre localement bornée et $c_{n} \in A_{n}$, tels que $A_{n}$ et $c_{n}$ vérifient les conditions de notre lemme pour $p=p_{n-1}$. Soit \|\|$_{n}$ une $p_{n}$-norme sous-multiplicative définissant la topologie de $A_{n}$, en remplaçant l'élément $c_{n}$ par $\left(\frac{1}{\left\|c_{n}\right\|_{n}}\right)^{\frac{1}{p_{n}}} c_{n}$, on peut supposer que $\left\|c_{n}\right\|_{n}=1$.

Soit $B$ l'algèbre somme directe des algèbres $A_{n}, n \in \mathbf{N}$, qu'on note:

$$
B=\bigoplus_{n \in \mathbf{N}} A_{n} \quad\left(B=\left\{u=\left(u_{n}\right)_{n \in \mathbf{N}} \in \prod_{n \in \mathbf{N}} A_{n} / u_{n}=0 \text { pour } n \geq n_{u}\right\}\right)
$$

on munit $B$ de la F-norme sous-multiplicative $\|$ || définie par:

$$
\left\|\left(u_{n}\right)_{n \in \mathbf{N}}\right\|=\sum_{n=1}^{\infty}\left\|u_{n}\right\|_{n}
$$

Soit $M$ le sous-espace vectoriel de $B$ engendré par $\left\{c_{1}-c_{n} / n \in \mathbf{N}\right\}$ (nous identifions les algèbres $A_{n}$ à leurs images canoniques). L'espace $M$ est un idéal de $B$ et $c_{1} \notin \bar{M}$ (la fermeture de $M$ dans $B$ ). En effet, pour tout $u=\sum_{n=2}^{n_{u}} \lambda_{n}\left(c_{1}-c_{n}\right) \in$ $M,\left\|u-c_{1}\right\|=\left|1-\left(\sum_{n=2}^{n_{u}} \lambda_{n}\right)\right|^{p_{1}}+\sum_{n=2}^{n_{u}}\left|\lambda_{n}\right|^{p_{n}}$ donc

$$
\left\|u-c_{1}\right\| \geq \max \left\{1-\sum_{n=2}^{n_{u}}\left|\lambda_{n}\right|^{p_{1}}+\sum_{n=2}^{n_{u}}\left|\lambda_{n}\right|^{p_{n}}, \max _{2 \leq n \leq n_{u}}\left\{\left|\lambda_{n}\right|^{p_{n}}\right\}\right\} \geq 1 .
$$


Soit $A$ l'algèbre topologique quotient $B / \bar{M}$, et soit $c$ l'image de $c_{1}$ par la surjection canonique, évidement $c$ est l'image de $c_{n}(n \in \mathbf{N})$ par la surjection canonique et $c \neq 0$. L'algèbre $A$ ne peut être munie d'aucune topologie d'algèbre localementpseudoconvexe. Sinon, il existera pour un certain $p \in] 0,1]$ deux $p$-disques absorbant $V$ et $W$ vérifiant: $V \cdot V \subseteq W$ et $c \notin W$.

Soit $V^{\prime}$ (resp. $W^{\prime}$ ) l'image réciproque de $V$ (resp. $W$ ) par la surjection canonique, soit $n \in \mathbf{N}$ tel que $p_{n-1}<p$. Sur l'algèbre $A_{n}$ les $p_{n-1}$-disques absorbant $V^{\prime} \cap A_{n}$ et $W^{\prime} \cap A_{n}$ vérfient les conditions de notre lemme, donc $c_{n} \in W^{\prime}$, ainsi $c \in W$ ce qui est absurde. Ceci nous permet de déduire que l'algèbre complétée de l'algèbre topologique $A$ montre le théorème.

\section{REMERCIEMENT}

Nous remercions le rapporteur pour ses remarques utiles qui nous ont permis d'améliorer la version de ce papier.

\section{BIBLIOGRAPHIE}

[1] J. Esterle. Sur la métrisabilité de certaines algèbres d'opérateurs. Rev. Roumaine Math. Pures Appl. 24 (1979), pp. 1157-1164. MR 81c:46002

[2] V. Müller. On topologizable algebras. Studia. Math 99 (1991), pp. 149-153. MR 92k:46081

[3] S. Rolewicz. Metric linear spaces. PWN, Warzawa, 1972. MR 55:10993

[4] S. Simmons. Boundedness in linear topological spaces. Trans. Amer. Math. Soc. 113 (1964), pp. 169-180. MR 29:3863

[5] P. Turpin. Sur une classe d'algèbres topologiques généralisant les algèbres localement bornées, These, Univ de Grenoble, 1966. MR 34:6566

[6] W. Żelazko. Concerning topologization of real or complex algebras. Colloq. Math. Nol. 71 (1996), pp. 111-113. MR 97f:46070

[7] W. Żelazko. Example of an algebra which is nontopologizable as a locally convex topological algebra. Proc. Amer. Math. Soc 110 (1990), pp. 947-949. MR 91c:46068

[8] W. Żelazko. On the locally bounded and m-convex topological algebras, Studia. Math. 19 (1960), pp. 333-356. MR 23:A4033

Département de Mathématiques, Univérsité Mohammed V, Faculté des Sciences, B.P 1014 Rabat, Maroc

E-mail address: zinelab@hotmail.com

Département de Mathématiques, Univérsité Mohammed V, Faculté des Sciences, B.P 1014 Rabat, Maroc

E-mail address: chidami@fsr.ac.ma 